\title{
Idiopathic Orbital Myositis Associated with Graves’ Disease
}

\author{
Seigo Tachibana ${ }^{1,4}$, Tadao Yokoi $^{2}$, Shinya Sato ${ }^{2}$, Yumi Oda ${ }^{3}$, \\ Toshihiko Yanase ${ }^{4}$ and Hiroyuki Yamashita ${ }^{2}$
}

\begin{abstract}
A 52-year-old man was referred to our clinic. One week before his visit, he had complained of left eye pain and double vision. His clinical features were exacerbated. Despite the acute onset, which is atypical of thyroid eye disease (TED), TED was suspected due to the patient's history of Graves' disease (GD). After conducting clinical examinations and orbital magnetic resonance imaging, the patient was diagnosed with idiopathic orbital myositis (IOM), and intravenous glucocorticoid therapy was administered. After treatment, the patient's clinical manifestations dramatically improved. This is a rare case in that the history of GD made it difficult to differentiate IOM from TED.
\end{abstract}

Key words: idiopathic orbital myositis, Graves' disease, thyroid eye disease, glucocorticoid therapy

(Intern Med 52: 787-790, 2013)

(DOI: 10.2169/internalmedicine.52.9162)

\section{Introduction}

Idiopathic orbital myositis (IOM) is a benign idiopathic inflammatory disease that may affect any structure in the orbit (1). IOM is the third most common cause of orbital inflammation, accounting for $4.7 \%$ to $6.3 \%$ of cases. Associations with viral infections and systemic diseases have been reported and autoimmunity has been suggested; however, the true pathogenesis remains elusive (2). Inflammation of orbital tissue leads to proptosis, eye pain, double vision due to restricted eye movement and other symptoms reflecting inflammation such as eyelid swelling, eyelid erythema, conjunctival redness, chemosis and swollen caruncles. On the other hand, thyroid eye disease (TED) is an important pathology of Graves' disease (GD). It is common for GD patients to complain of discomfort associated with their eyes. The etiology of TED also involves inflammation and fibrosis of the extraocular muscles (EOMs) and/or proliferation of orbital fatty tissue. This etiology also leads to clinical features similar to IOM. When endocrinologists see TED patients, it is important for them to differentiate TED from other orbital diseases such as IOM, caroticocavernous fistulae, non-Hodgkin's lymphoma and orbital meningioma (3).
In this paper, we report a rare case of IOM associated with GD, focusing on its diagnosis and treatment.

\section{Case Report}

A 52-year-old man was referred to our clinic due to suspicion of TED. When he was 47 years old, he was diagnosed with GD owing to thyrotoxicosis with diffuse goiter and a high titer of TSH receptor antibodies (TRAb). When he was diagnosed with GD, he had no complaints associated with TED. After the diagnosis, the patient received methimazole (MMI) continuously for five years, and his thyroid function was stable as a result of MMI at a dose of $5 \mathrm{mg}$ per day for over two years. One week before he visited our clinic, the patient complained of left eye pain and double vision. Subsequently, his symptoms worsened: eyelid swelling, conjunctival redness, chemosis, eyelid erythema and ptosis presented in the left eye. He visited an ophthalmologist, and a diagnosis of TED was suspected due to his history of GD. On the first visit, the patient was found to be $172.0 \mathrm{~cm}$ tall and weighed $68.5 \mathrm{~kg}$, with a body mass index of 23.2. His blood pressure was $122 / 70 \mathrm{mmHg}$ and his pulse rate was 66 beats/min and regular. His clinical activity score (CAS) was 6/7: spontaneous eye pain, eyelid swelling, eyelid erythema,

\footnotetext{
${ }^{1}$ Department of Endocrinology, Yamashita Thyroid and Parathyroid Clinic, Japan, ${ }^{2}$ Department of Surgery, Yamashita Thyroid and Parathyroid Clinic, Japan, ${ }^{3}$ Department of Ophthalmology, Hayashi Eye Hospital, Japan and ${ }^{4}$ Department of Endocrinology and Diabetes Medicine, Fukuoka University Hospital, Japan

Received for publication October 19, 2012; Accepted for publication December 25, 2012

Correspondence to Dr. Seigo Tachibana, sei.tac.thyroid@gmail.com
} 
Table 1. Laboratory Data

\begin{tabular}{l}
\hline Blood count \\
\hline WBC $9,300 / \mu \mathrm{L}$, RBC $520 \times 10^{4} / \mu \mathrm{L}, \mathrm{Hb} 16.1 \mathrm{~g} / \mathrm{dL}, \mathrm{Hct} 47.3 \%$, Plt $20.4 \times 10^{4} / \mu \mathrm{L}$ \\
Lym $19.1 \%$, Neu $69.4 \%$ \\
\hline Blood chemistry \\
\hline TP $7.3 \mathrm{~g} / \mathrm{dL}$, Alb $4.5 \mathrm{~g} / \mathrm{dL}$, AST $19 \mathrm{IU} / \mathrm{L}$, ALT $20 \mathrm{IU} / \mathrm{L}, \mathrm{ALP} 161 \mathrm{IU} / \mathrm{L}, \mathrm{CPK} 63 \mathrm{IU} / \mathrm{L}$ \\
Cre $0.9 \mathrm{mg} / \mathrm{dL}, \mathrm{T}-\mathrm{Chol} 228 \mathrm{mg} / \mathrm{dL}$, glucose $105 \mathrm{mg} / \mathrm{dL}$ \\
Na $140 \mathrm{mEq} / \mathrm{L}, \mathrm{K} 3.4 \mathrm{mEq} / \mathrm{L}, \mathrm{Cl} 102 \mathrm{mEq} / \mathrm{L}, \mathrm{Ca} 9.8 \mathrm{mg} / \mathrm{dL}, \mathrm{IP} 3.2 \mathrm{mg} / \mathrm{dL}$ \\
\hline Endocrinological findings \\
\hline TSH $5.55 \mu \mathrm{IU} / \mathrm{mL}$, free thyroxine $1.35 \mathrm{ng} / \mathrm{dL}$, free triiodothyronine $3.96 \mathrm{pg} / \mathrm{mL}$, \\
TRAb $(3 \mathrm{rd}$ generation) $0.6 \mathrm{IU} / \mathrm{L}, \mathrm{TSAb} 121 \%, \mathrm{TgAb} 18.4 \mathrm{IU} / \mathrm{mL}, \mathrm{TPOAb} 21.0 \mathrm{IU} / \mathrm{mL}$ \\
\hline
\end{tabular}
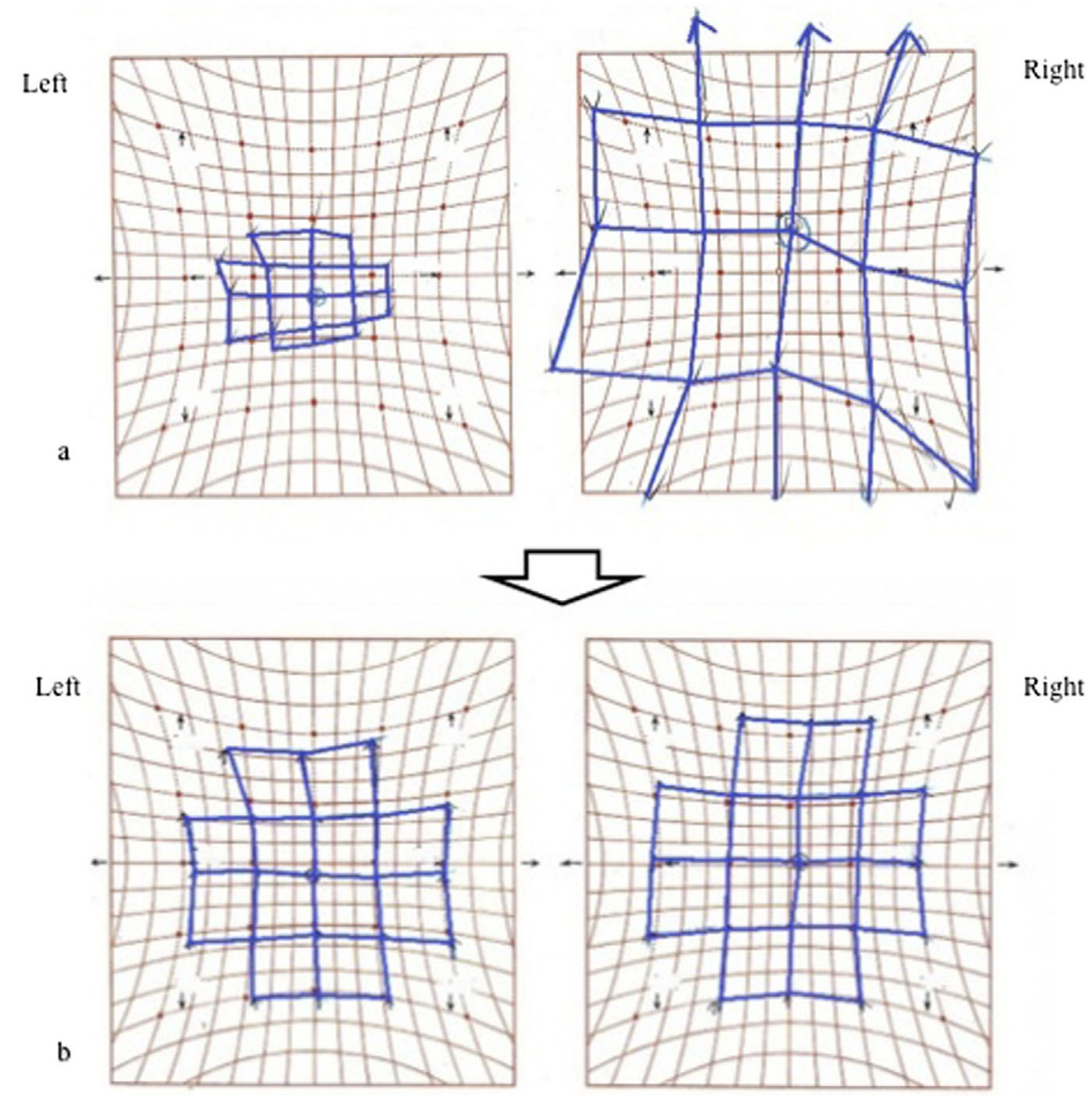

Figure 1. a. Hess screen test before treatment showed severe restriction of left eye movement in all directions. b. Hess screen test after treatment showed significant improvement of left eye movement.

conjunctival redness, chemosis and a swollen caruncle. In addition, he had severe ptosis of the left eye without lid retraction. His right fractional visual acuity was 20/20, and his left fractional visual acuity was 20/40. His intraocular pressure was as follows: right eye: $16 \mathrm{mmHg}$, left eye: 17 $\mathrm{mmHg}$. The measurement of proptosis using a Hertel proptometer was as follows: right: $17 \mathrm{~mm}$, left: $17 \mathrm{~mm}$. The patient's thyroid was diffusely palpable; however, other physical examinations showed normal results. He had a history of hyperuricemia without medication use and partial hepatectomy for a liver transplant to his mother. His family history revealed maternal thyroid disease. He had smoked a pack of cigarettes a day for 30 years and did not drink alcohol.

The patient's laboratory data are shown in Table 1. His thyroid function while taking MMI at a dose of $5 \mathrm{mg}$ per day revealed subclinical hypothyroidism. All levels of thyroid autoantibodies, except TPOAb, were normal. The patient's left eye motion was severely restricted, as determined by a Hess screen test (Fig. 1a). Orbital magnetic resonance (MR) imaging showed a remarkably swollen left superior rectus muscle (SRM) (Fig. 2a). The swollen EOM involved only the left SRM and the tendon. The border of the SRM was irregular due to inflammation, and fat noise was also observed. A diagnosis of IOM, not TED, was suspected due to the relatively acute clinical course and the clinical signs and MR imaging results atypical of TED. Other orbital diseases such as caroticocavernous fistulae, non-Hodgkin's lymphoma and orbital meningioma were less likely. There- 

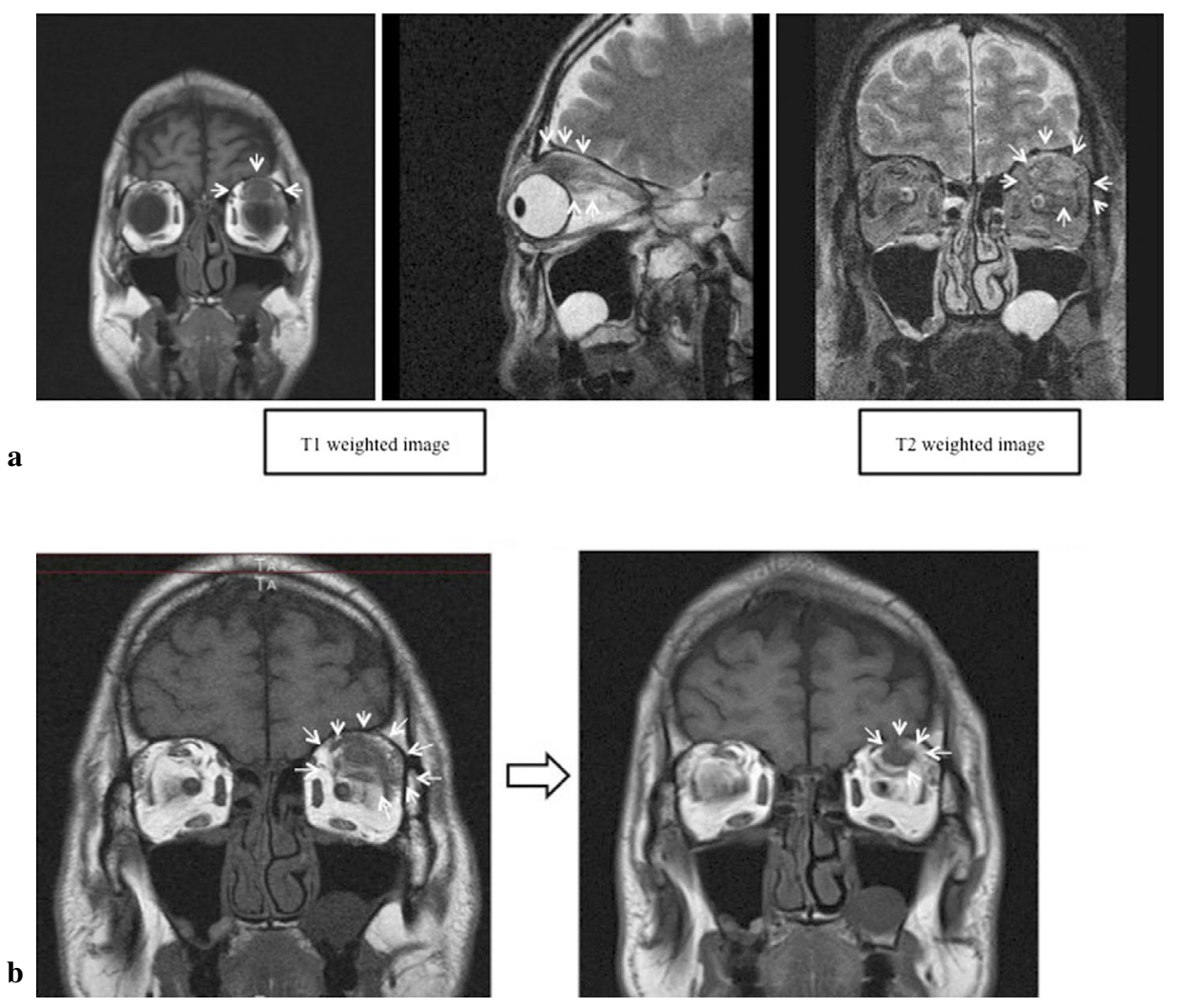

Figure 2. a. Orbital MR imaging before treatment showed severe SRM swelling and long T2 relaxation times with an irregular border of SRM and fat noise. The tendon of SRM was also involved. b. Orbital MR imaging after treatment showed significant improvement.

fore, the patient was diagnosed as having IOM associated with GD.

Intravenous glucocorticoid pulse therapy (IVGC) with methylprednisolone (mPSL) was initiated. A daily dose of $1,000 \mathrm{mg}$ of $\mathrm{mPSL}$ was prescribed three times a week, then oral PSL was administered. After two courses of IVGC, the patient's clinical features improved dramatically. Two months after treatment, the CAS was reduced to $0 / 7$, and the Hess screen test and orbital MR imaging findings were also remarkably improved, as shown in Figs. 1b, 2b. In addition, the ophthalmological findings were improved as follows: the right fractional visual acuity was $20 / 25$, and the left fractional visual acuity was 20/22. The patient's intraocular pressure was as follows: right eye: $14 \mathrm{mmHg}$, left eye: 16 $\mathrm{mmHg}$. The measurement of proptosis using a Hertel proptometer was as follows: right: $17 \mathrm{~mm}$, left: $17 \mathrm{~mm}$. After treatment, remission of IOM was maintained.

\section{Discussion}

This was a rare case of IOM associated with GD. Orbital pseudotumors, first described by Gleason in 1903 and later named by Birch-Hirschfield in 1930, are a benign idiopathic inflammatory disease that may affect any structure in the orbit (1). IOM represents a subgroup within idiopathic orbital inflammatory syndrome, formerly termed orbital pseudotumor. IOM describes a rare inflammatory disorder of EOMs (4). The clinical manifestations of IOM are as fol- lows: eye pain, diplopia, proptosis, eyelid swelling, eyelid erythema, chemosis, conjunctival redness, swollen caruncles and ptosis. These clinical manifestations are exhibited acutely or subacutely. The pathogenesis of IOM is not completely understood; however, an immune-mediated process has been considered (5). The disease has been found to be associated with infection, trauma and surgery. The clinical diagnosis of IOM is made by excluding other conditions. Orbital MR and CT imaging are useful for making a diagnosis of orbital disease (4). In patients with IOM, the typical features of MR imaging include unilateral enlargement of the EOM, an irregular border of the EOM with fat noise and involved tendons of the EOM (3). These MR imaging features are very important for differentiating IOM from TED. The differences in clinical and MR imaging features are summarized in Table 2 (3).

Due to the patient's history of GD, a diagnosis of TED was initially suspected in this case. There is a case report of severe TED in which only $\mathrm{TgAb}$ and TPOAb were detected, not TRAb and TSAb (6). A diagnosis of TED could not be excluded based on the negative results of TRAb and TSAb in our case. The acute clinical course and features observed in this case, particularly severe eye pain and ptosis, are not typical of TED. In general, TED exhibits a relatively chronic onset (weeks to months), mild eye pain and lid retraction. On the other hand, IOM exhibits acute onset (days), severe eye pain and ptosis, not lid retraction (3). In addition, orbital MR imaging showed the above-mentioned 
Table 2. The Differences in Clinical and MR Imaging Features

\begin{tabular}{|c|c|c|}
\hline & IOM & $\mathrm{GO}$ \\
\hline \multicolumn{3}{|l|}{ [Clinic] } \\
\hline Onset & days & weeks to months \\
\hline Pain & severe & mild \\
\hline Eye lid position & $\begin{array}{l}\text { ptosis frequent / } \\
\text { retraction rare }\end{array}$ & retraction frequent \\
\hline $\begin{array}{l}\text { Steroid response } \\
\text { [Imaging] }\end{array}$ & usually complete and fast & incomplete and slow \\
\hline Bilateral & sometimes & frequently \\
\hline EOM involoved & & \\
\hline Multiple EOM are involved & rarely & usually \\
\hline EOM borders & irregular, fat noise & regular, no fat noise \\
\hline Tendon involved & yes & no \\
\hline
\end{tabular}

features suggestive of IOM. Therefore, the patient was diagnosed with IOM.

The first-line therapy for IOM is glucocorticoid therapy $(2,4,7)$. It is well known that oral glucocorticoid therapy results in prompt improvement and remission within days to weeks in most patients. However, despite the good response to oral glucocorticoid therapy exhibited by IOM, it is also known that remission of IOM is often incomplete and recurrence is frequently observed. In severe cases, IVGC is effective; however, Bijlsma et al. suggested that the indications for IVGC are limited to accelerating symptom relief and recovery from optic nerve dysfunction, as there are no significant differences in the total dose of glucocorticoids, the duration of treatment or decreases in persistent symptoms between IVGC and oral glucocorticoid therapy (8). On the other hand, the first-line therapy for moderate to severe TED is also IVGC $(9,10)$. However, unlike the treatment for IOM, IVGC for TED does not show prompt improvement and remission. In addition, in moderate to severe TED patients with restricted eye movement, retrobulbar irradiation $(\mathrm{RBI})$ is recommended $(3,11,12)$. In IOM patients, the indications for RBI are limited to cases that show no clinical improvements after glucocorticoid therapy, glucocorticoid dependence or serious adverse effects of glucocorticoids (5). Therefore, it is important to differentiate IOM from TED because the management and prognosis after treatment of these diseases differ. In this case, after the diagnosis of IOM with severe clinical features and rapid exaggeration of eye symptoms, IVGC therapy was initiated and prompt improvement of the patient's clinical features was observed. The patient's clinical course after treatment was also compatible with a diagnosis of IOM.

This was a rare case of IOM with GD. According to a re- view of the literature, there are no case reports of IOM in GD patients. In general, when a patient with proptosis has a history of GD, a diagnosis of TED is first suspected. Therefore, in this case, the history of GD made it difficult to differentiate IOM from TED. We considered this case to be an important case associated with the differential diagnosis of proptosis. In conclusion, it is important to recognize the clinical features of IOM and TED because TED cannot be excluded based only on an immunological examination associated with GD.

The authors state that they have no Conflict of Interest (COI).

\section{References}

1. Scott IU, Siatkowski RM. Idiopathic orbital myositis. Curr Opin Rheumatol 9: 504-512, 1997.

2. Lutt JR, Lim LL, Phal PM, Rosenbaum JT. Orbital inflammatory disease. Semin Arthritis Rheum 37: 207-222, 2008.

3. Mourits MP. Diagnosis and Differential Diagnosis of Graves' Orbitopathy. In: Graves' Orbitopathy: A Multidisciplinary ApproachQuestions and Answers. 2nd ed. Wiersinga WM, Kahaly GJ, Eds. Karger, Basel, 2010: 66-76.

4. Schoser BG. Ocular myositis: diagnostic assessment, differential diagnoses, and therapy of a rare muscle disease: five new cases and review. Clin Ophthalmol 1: 37-42, 2007.

5. Caminha LS, Pinto ER, Sousa PA, Oliveira RA, Conceicao FL, Vaisman M. Orbital pseudotumor: a differential diagnosis of Graves' ophthalmopathy. Arq Bras Endocrinol Metab 55: 85-88, 2011.

6. Yoshihara A, Yoshimura Noh J, Nakachi A, et al. Severe thyroidassociated orbitopathy in Hashimoto's thyroiditis. Report of 2 cases. Endocr J 58: 343-348, 2011.

7. Yuen SJ, Rubin PA. Idiopathic orbital inflammation: distribution, clinical futures, and treatment outcome. Arch Ophthalmol 121: 491-499, 2003.

8. Bijlsma WR, Paridaens D, Kalmann R. Treatment of severe idiopathic orbital inflammation with intravenous methylprednisolone. Br J Ophthalmol 95: 1068-1071, 2011.

9. Bartalena L, Baldeschi L, Dickinson A, et al. Consensus statement of the European Group on Graves' orbitopathy (EUGOGO) on management of GO. Eur J Endocrinol 158: 273-285, 2008.

10. Stiebel-Kalish H, Robenshtok E, Hasanreisoqlu M, Ezrachi D, Shimon I, Leibovici L. Treatment modalities for Graves' ophthalmopathy: systematic review and metaanalysis. J Clin Endocrinol Metab 94: 2708-2716, 2009.

11. Bradley EA, Gower EW, Bradley DJ, et al. Orbital radiation for graves ophthalmopathy: a report by the American Academy of Ophthalmology. Ophthalmology 115: 398-409, 2008.

12. Zouamlan CI, Cockerham KP, Turbin RE, et al. Efficacy of corticosteroids and external beam radiation in the management of moderate to severe thyroid eye disease. J Neuroophthalmol 27: 205-214, 2007.

(C) 2013 The Japanese Society of Internal Medicine http://www.naika.or.jp/imonline/index.html 